\title{
Auch bei hohem Reintubationsrisiko effektiv
}

Fragestellung: Ziel dieser Studie war es, die High-flow-Sauerstofftherapie über eine spezielle Nasensonde mit der herkömmlichen Variante, der nicht invasiven Beatmung (NIV), bei Patienten mit hohem Risiko einer Reintubation hinsichtlich einer Nichtunterlegenheit zu überprüfen.

Hintergrund: Nach einer längeren Intubation ist oft zunächst die Gabe von Sauerstoff nötig, um eine ausreichende Sauerstoffsättigung zu erreichen. Hierfür stehen verschiedene Methoden zur Verfügung: Zuführung von Sauerstoff über eine herkömmliche Sauerstoffmaske, Anwendung der NIV über eine spezielle Atemmaske sowie die seit mehreren Jahren verfügbare High-flow-Sauerstofftherapie über eine spezielle Nasensonde. Vergleichende Untersuchungen fehlten bisher.

Patienten und Methodik: Die randomisierte Studie wurde auf drei Intensivstationen durchgeführt. Einschlusskriterien waren eine Beatmungsdauer von mindestens zwölf Stunden

Hernandez G, Vaquero C, Colinas $L$ et al. Effect of postextubation high-flow nasal cannula vs noninvasive ventilation on reintubation and postextubation respiratory failure in high-risk patients: A randomized clinical trial. JAMA 2016; 316: 1565 - 74 sowie das erfolgreiche Durchlaufen eines speziellen Protokolls zur Entwöhnung von der Beatmung. Zudem mussten die Patienten bestimmte Kriterien erfüllen, die sie als eine Hochrisikogruppe für eine Reintubation kennzeichneten (hohes Patientenalter, chronische obstruktive Lungenerkrankung [COPD], Herzinsuffizienz, große Anzahl an Vorerkrankungen, hohes Körpergewicht oder Langzeitbeatmung über sieben Tage). Die Patienten wurden dann entweder in die NIV- oder in die High-flow-Sauerstofftherapiegruppe randomisiert. Die Behandlung erfolgte über 24 Stunden, danach wurde auf eine herkömmliche Sauerstofftherapie mit Maske gewechselt. Der primäre Endpunkt umfasste die Reintubation innerhalb von 72 Stunden sowie das Auftreten einer respiratorischen Insuffizienz.

Ergebnisse: In die Studie wurden insgesamt 604 Patienten eingeschlossen und randomisiert. Die Reintubationsrate nach 72 Stunden war zwischen der NIV- und der High-flow Sauerstofftherapiegruppe nicht unterschiedlich (19,1\% vs. $22,8 \%)$. Patienten mit NIV entwickelten häufiger eine respiratorische Insuffizienz als Patienten mit High-flow-Sauerstofftherapie (39,8\% vs. $26,9 \%)$. Der durchschnittliche Aufenthalt auf der Intensivstation war kürzer in der Gruppe der mit High-flowSauerstofftherapie behandelten Patienten (drei Tage vs. vier Tage, $p=0,048$ ). Alle Patienten zeigten eine gute Toleranz der High-flow-Sauerstofftherapie, die durchschnittliche Zeit der NIV war 14 Stunden.

Schlussfolgerungen: Die Anwendung der High-flow-Sauerstofftherapie ist der NIV auch bei Patienten mit einem hohen Risiko für eine Reintubation nicht unterlegen.

\section{- Kommentar von Tobias Bobinger und Hagen B. Huttner, Erlangen}

\section{Auch bei Risikopatienten so wirksam wie die üblichen Methoden}

In dieser Arbeit wurde erstmals eine High-flow-Sauerstofftherapie mit der herkömmlichen NIV an einem Kollektiv von Hochrisikopatienten verglichen und die Nichtunterlegenheit dieser Methode im Hinblick auf die Vermeidung von Reintubation und respiratorischer Insuffizienz demonstriert. Anzumerken ist, dass die Therapie von allen Patienten gut toleriert wurde, und auch keine Unterschiede in den sekundären Endpunkten wie etwa Infekt, Sepsis oder Mortalität beobachtet wurden. Verglichen mit anderen Studien mit NIV zeigte sich hier eine höhere Rate an Reintubationen in der NIV-Gruppe. Dies ist jedoch neben dem Risikoprofil der Patienten zum einen durch die kurze Anwendungsdauer der NIV von nur 24 Stunden zu erklären, zum anderen waren Sedativa zur Verbesserung der Toleranz im Studiendesign nicht erlaubt. Insbesondere in der Gruppe der Highflow-Sauerstofftherapie zeigte sich eine deutliche Zunahme der Reintubationen nach 24 Stunden, genau zu dem Zeitpunkt, als in der Studie von dieser Therapie auf eine konventionelle Sauerstoffapplikation mit Maske gewechselt wurde. Eine Ausdehnung der Anwendung zum Beispiel auf 48 Stunden könnte - wie in anderen Studien gezeigt - eine Reintubation in dieser Gruppe verhindern. Die Durchführung dieser Studie erfolgte auf einer allgemeinen Intensivstation. Etwa ein Viertel der Patienten (23,2\% vs. $28,6 \%$ ) wies ein neurologisches Krankheitsbild auf. Neuromuskuläre Erkrankungen wie beispielsweise Myasthenia gravis oder Guillain-Barré-Syndrom stellen eine Herausforderung in der Entwöhnung vom Beatmungsgerät sowie der anschließenden Extubationsphase dar. Die einfache Anwendbarkeit und gute Toleranz der High-flow-Sauerstofftherapie eröffnet eine neue Möglichkeit, deren Nichtunterlegenheit gegenüber herkömmlichen Methoden durch diese Studie eindrucksvoll bestätigt wird.

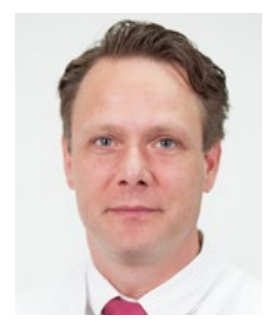

Prof. Dr. med. Hagen B. Huttner, Erlangen

Universitätsklinik für Neurologie, Erlangen E-Mail: hagen.huttner@uk-erlangen.de 